\title{
Liver Fibrosis Assessment Using Transient Elastography by FibroScan and Shear Wave Elastography by Sonography: A Comparative Cross-sectional Study in an Outpatient Liver Clinic
}

\author{
Fariba Zarei ${ }^{1,2}$, Maryam Moini ${ }^{3}$, Mahsa Abedi ${ }^{2}$, Rezvan Ravanfar Haghighi ${ }^{1}$ and Banafsheh \\ Zeinali-Rafsanjani ${ }^{1,{ }^{*}}$ \\ ${ }^{1}$ Medical Imaging Research Center, Shiraz University of Medical Sciences, Shiraz, Iran \\ ${ }^{2}$ Department of Radiology, Shiraz University of Medical Sciences, Shiraz, Iran \\ ${ }^{3}$ Gastroenterohepatology Research Center, Shiraz University of Medical Sciences, Shiraz, Iran \\ "Corresponding author: Medical Imaging Research Center, Shiraz University of Medical Sciences, Shiraz, Iran. Email: b.zeinali.r@gmail.com
}

Received 2020 December 29; Revised 2021 September 17; Accepted 2021 September 19.

\begin{abstract}
Background: Non-alcoholic fatty liver disease (NAFLD) is the second most common cause of liver transplantation in the United States, with a continuously growing prevalence. There are several non-invasive methods to detect liver fibrosis, which is defined as the accumulation of extracellular matrix proteins, particularly collagens. It is most commonly associated with chronic liver diseases, such as NAFLD.

Objectives: This study aimed to investigate the concordance between transient elastography (TE) and shear wave elastography (SWE) for liver fibrosis staging and also to examine the congruence between the controlled attenuation parameter (CAP) and the B-mode hepatorenal ratio for hepatic steatosis grading in patients with NAFLD.

Patients and Methods: In this cross-sectional study conducted during March 2018 - 2019, NAFLD patients, referred to the liver clinic of our center for the non-invasive assessment of hepatic fibrosis, were enrolled. However, patients with sonographic features of cirrhosis, multiple hepatic masses, or moderate to large ascites were excluded; also, patients who were uncooperative during the tests were excluded. Measurements obtained by different tools were recorded. Kolmogorov-Smirnov test, Chi-square test, independent $t$-test, or Mann-Whitney tests, as well as Pearson's correlation coefficient test, were used to analyze the data.

Results: Sixty-five patients (male-to-female ratio, 1:13), with a median age of 47 years, were included in the study. The tools for assessing fibrosis ( $\mathrm{r}=0.9538,95 \%$ CI: 0.9252 - 0.9717, P < 0.0001) and steatosis ( $\mathrm{r}=0.429,95 \%$ CI: 0.2048 - $0.6104, \mathrm{P}<0.0001)$ were perfectly and moderately correlated, respectively. Sex, age, and body mass index (BMI) did not affect the results.

Conclusion: The two elastography modalities showed a strong correlation for fibrosis staging in our study population. Also, the CAP and B-mode hepatorenal ratio were moderately correlated for grading hepatosteatosis. Overall, selection of the best assessment method among the studied modalities depends on factors other than internal validity.
\end{abstract}

Keywords: Liver Fibrosis, Fibroscan, Shear Wave, Transient Elastography

\section{Background}

Chronic liver disease, which is known to have various etiologies, can result in hepatic fibrosis. The prevalence of non-alcoholic fatty liver disease (NAFLD) is estimated at $25 \%$. NAFLD is rapidly becoming a major cause of chronic liver disease (1). It has been reported as the second most common cause of liver transplantation in the United States, with an ongoing increase in prevalence.(2)

Liver fibrosis is defined as the accumulation of extracellular matrix proteins, particularly collagen, which is most commonly associated with chronic liver diseases (3). Hepatic steatosis is described as the accumulation of fat within the cytoplasm of hepatocytes $(4,5)$. The most effective treatment for hepatic fibrosis is to manage the underlying cause of liver disease. Besides, antifibrotic treatments targeting hepatic stellate cells, as progenitors of hepatic fibrosis, and different key cytokines involved in fibrogenesis have been highlighted in different studies (6).

The main factor in the management of patients with chronic liver disease is monitoring hepatic fibrosis for progression or possible regression in case of effective treatment. Diagnosis in earlier stages is also crucial for management and improved outcomes. Liver biopsy, as the gold standard for the assessment of hepatic fibrosis, is an inva- 
sive method, associated with some complications (7, 8). Accordingly, non-invasive, reliable, simple, and cost-effective methods are needed. Transient elastography (TE) using a FibroScan system and shear wave elastography (SWE) using sonography have been introduced for evaluating fibrosis, while the controlled attenuation parameter (CAP) measured by FibroScan and the B-mode hepatorenal ratio measured by sonography have been introduced for evaluating hepatosteatosis (9-12).

\section{Objectives}

This study aimed to examine the concordance between different tools used for measuring liver fibrosis (TE vs. SWE) and hepatosteatosis (CAP vs. B-mode ratio) and to determine the effects of demographic variables on these measurements in patients, referred to the liver clinic of our university-affiliated center.

\section{Patients and Methods}

\subsection{Participants}

In this cross-sectional study, NAFLD patients, referred to the liver clinic of our center for a non-invasive assessment of hepatic fibrosis during March 2018 - 2019, were enrolled. The patients consented to undergo two methods of fibrosis and steatosis assessment (TE and CAP measurements by FibroScan and SWE and B-mode sonography). It should be noted that written consent was obtained from each participant before participation in the study. This study was conducted based on the Declaration of Helsinki. The university ethics committee approved the study protocol (ethics code: IR.sums.med.rec.1397.454). Patients with sonographic features of cirrhosis (ie, an irregular liver surface and lobar redistribution), multiple hepatic masses, or moderate to large ascites, as well as uncooperative patients during the tests, were excluded.

\subsection{Procedures and Assessments}

FibroScan was performed by a gastroenterologist in the liver clinic. In a supine position, the patient's right arm was held upright to access the right hypochondrium. The optimal place for the probe was where the midaxillary vertical line crossed the xiphoidal horizontal line. A $5-\mathrm{MHz}$ unidimensional M-probe was inserted $25-65 \mathrm{~mm}$ beneath the skin over a $4 \times 1 \mathrm{~cm}$ region of interest (ROI); the mentioned method was repeated ten times. The median fibrosis (elasticity) score was presented in kilopascal (kPa) and classified based on the METAVIR score: F0-1, 5.5 (4.1 - 7.1) kPa; F2, 6.6 (4.8 - 9.6) kPa; F3, 10.3 (7.6 - 12.9) kPa; and F4, 30.8 (16.3 - 48) $\mathrm{kPa}$. Moreover, the Brunt steatosis staging system was applied according to the percentage of fat accumulation in hepatocytes (S0, 0 - 10\%; S1, 11 - 33\%; S2, 34 - 66\%; and S3, $67-100 \%)$, in addition to the median steatosis CAP score in $\mathrm{dB} / \mathrm{m}$.

SWE was performed by a staff radiologist using a SuperSonic device in the radiology clinic. The patients were instructed to fast for at least six hours. While they were in the left lateral position, their right arm was abducted. They were asked to hold their breath for at least six seconds for placing a 7-MHz concave probe on the right intercostal space. The best place for the probe was over the right lobe of the liver, where there was no sign of a large vessel or lesion, with an appropriate tissue resolution (distance to hepatic capsule $\geq 2 \mathrm{~cm}$ ).

The fibrosis stage was measured after a homogeneous color mapping. This method was repeated ten times, and the median fibrosis (elasticity) score was recorded in $\mathrm{kPa}$. The second heapatosteatosis assessment was the B-mode hepatorenal ratio measurement, which classified the patients into three groups: mild, $<1.49 ; 1.49 \leq$ moderate $<$ 1.86; and severe, $\geq 1.86$. Besides, the conventional grayscale sonographic data and demographic information, including the body mass index (BMI), sex, and age, were recorded for every patient.

\subsection{Data Analysis}

Statistical analysis was performed using SPSS Version 22.0 for Windows (released in 2013, IBM Corp., Armonk, NY, USA), as well as the Deducer Package powered by R programming language (Version 3.4.4 for Windows). Qualitative and quantitative variables were described using frequency (percentage) and mean \pm standard deviation (SD) or median and interquartile range (ICR), respectively. They were also visualized by box plots and scatter plots.

Kolmogorov-Smirnov test was used to examine the distribution of variables, and non-parametric tests were used when there was no normal distribution. The groups were compared using Chi-square test for qualitative variables (male vs. female) and independent $t$-test or Mann-Whitney test for quantitative variables (BMI $<30 \mathrm{~kg} / \mathrm{m}^{2}$ vs. BMI $\geq$ $30 \mathrm{~kg} / \mathrm{m}^{2}$ ). Moreover, Pearson's correlation coefficient test showed a linear correlation between the measurements of different quantitative methods for fibrosis and steatosis grading. P-value $\leq 0.05$ was considered statistically significant.

\section{Results}

This study was performed on 65 patients, with the median age of 47 years (range, 41 - 55 years) and a male-tofemale ratio of 1:13. The missing data were excluded from 
the study. The mean BMI of the patients was $27.3 \mathrm{~kg} / \mathrm{m}^{2}$ (range: $23.6-29.6 \mathrm{~kg} / \mathrm{m}^{2}$ ), suggesting that most of the patients were overweight. The patients' demographic characteristics and the median TE, SWE, CAP, and B-mode ratio are presented in Table 1 and Figure 1.

\begin{tabular}{|c|c|}
\hline Variables & Values \\
\hline Number & 65 \\
\hline Age, median (ICR) & $47(41-55)$ \\
\hline \multicolumn{2}{|l|}{ Sex, No. (\%) } \\
\hline Female & $30(46.2)$ \\
\hline Male & $34(52.3)$ \\
\hline Missing & $1(1.5)$ \\
\hline BMI, median (ICR) & $27.3(23.6-29.6)$ \\
\hline TE, median kPa (ICR) & $6.1(5.1-7.6)$ \\
\hline SWE, median kPa (ICR) & $6.1(5.2-7.3)$ \\
\hline CAP, median dB/m (ICR) & $279(227.5-313.25)$ \\
\hline B-mode ratio, median score (ICR) & $1.44(1.17-1.86)$ \\
\hline
\end{tabular}

Abbreviations: BMI, body mass index; CAP, controlled attenuation parameter; ICR, interquartile range; SWE, shear wave elastography; TE, transient elastography.

The fibrosis staging tools, TE, and SWE yielded perfectly correlated measurements $(r=0.9538$; $95 \%$ confidence interval (CI): 0.9252 - 0.9717) (Figure 2A). Overall, 54 (83.1\%) patients had fibrosis scores $\leq 2$. The CAP and B-mode ratio were moderately correlated for steatosis grading $(r=0.429$; 95\% CI: 0.2048 - 0.6104) (Figure 2B), especially for moderate and severe hepatosteatosis. Compared to CAP and grayscale sonography, the B-mode ratio classified a greater proportion of the participants into mild steatosis (Table 2). There was no significant correlation between other variables (ie, sex, age, and BMI) and different staging tools ( $\mathrm{P}>$ 0.05). Nevertheless, a mild correlation was found between BMI and CAP $(\mathrm{P}=0.005)$ (Table 3 ).

\begin{tabular}{lccc}
\hline \multicolumn{4}{l}{ Table 2. Hepatic Steatosis Grades According to Different Tools ${ }^{\text {a }}$} \\
\hline Hepatic steatosis & $\begin{array}{c}\text { CAP }(\text { Brunt } \\
\text { score })\end{array}$ & $\begin{array}{c}\text { Gray-scale } \\
\text { sonography }\end{array}$ & B-mode ratio \\
\hline Steatosis grade & $16(24.6)$ & $15(23.1)$ & $6(9.2)$ \\
\hline So & $8(12.3)$ & $8(12.3)$ & $21(32.3)$ \\
S1 & $20(30.8)$ & $29(44.6)$ & $17(26.2)$ \\
S2 & $21(32.3)$ & $13(20)$ & $21(32.3)$ \\
\hline
\end{tabular}

Abbreviation: CAP, controlled attenuation parameter.

${ }^{a}$ Values are expressed as Number (percentage)

\section{Discussion}

Although liver biopsy is the gold standard for hepatic fibrosis staging, it is an invasive method, associated with rare, but serious potential risks of bleeding, damage to the adjacent organs, and even death due to complications. Besides, it is not the most cost-effective option, and resources, as well as expertise, are needed for sampling. Also, an expert pathologist must be present for the interpretation of histological findings. Moreover, liver biopsy is subject to sampling errors, as histological assessment is performed on a very small liver volume $(1 / 50,000)$; also, because the liver may not be homogenously involved in pathology, the assessment may not be precise (13). Therefore, with a global increase in the prevalence of chronic liver diseases, reliable and non-invasive methods of liver disease staging are needed.

\subsection{Fibrosis}

In the present study, the two non-invasive methods of TE and SWE were strongly correlated for fibrosis staging. This finding is consistent with several previous studies, including those conducted by Bende et al. $(r=0.83)(14)$, Kircheis et al. $(r=0.92)(15)$, Zeng et al. $(r=0.835)(16)$, and Paul et al. $(r=0.33)(17)$. Overall, there is no preference for the use of these two methods in fibrosis assessment, and the choice depends on factors unrelated to internal validity, including the operator's experience, cost-effectiveness, and availability (18). However, it seems that SWE has several advantages over TE.

SWE is a numeric and color-coded modality, in which the operator can choose the most homogenous ROI. Besides, it is a real-time modality; therefore, large vessels and the liver capsule can be avoided (14). In this regard, a meta-analysis by Bota et al. (19) showed that SWE fails less than TE (2.1\% vs. 6.6\%) in providing reliable measurements. Besides, the SWE results are more independent of steatosis than TE (20-24). Also, SWE is more available than TE. Because it is performed by an ultrasound machine, hepatosteatosis measurements based on gray-scale sonography or B-mode hepatorenal ratio can be performed simultaneously with the assessment of hepatobiliary imaging features. However, it should be noted that SWE is operatordependent and should be performed by a trained ultrasonographer; besides, the patient's cooperation is essential, and the examination time is longer.

A high BMI $\left(>28 \mathrm{~kg} / \mathrm{m}^{2}\right)$ or a small intercostal space can affect both TE and SWE results $(25,26)$. In the present study, the effect of BMI on the measurements was not significant, which is similar to the finding reported by Cassinotto et al. (27). Similar to our results, in another study, Cassinotto et al. (28) showed that SWE and TE results were not affected 

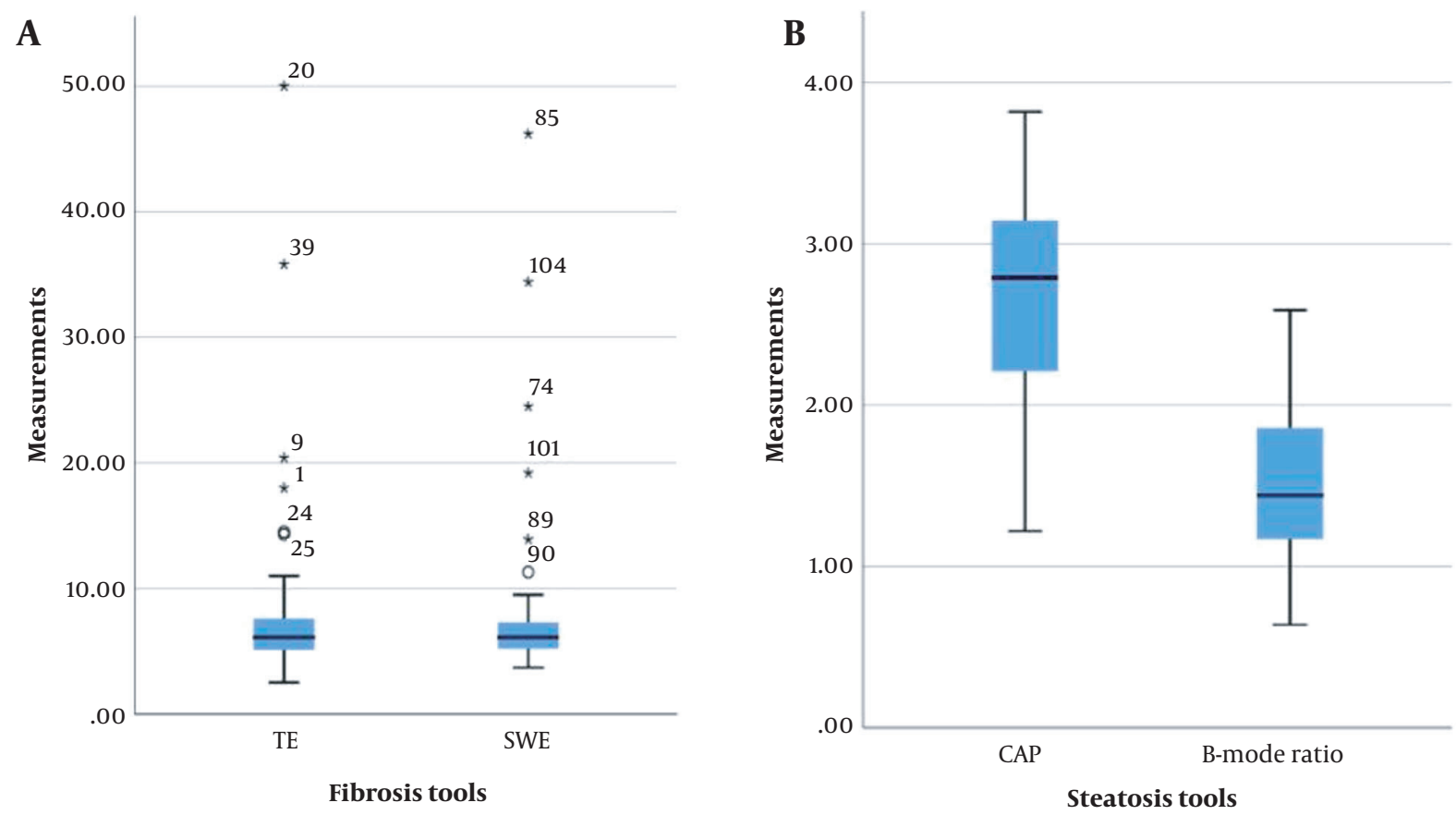

Figure 1. The box plot of median measurements of fibrosis (A) and steatosis (B) (central horizontal line: Median; upper and lower horizontal lines: First and third quartiles and corner horizontal lines: minimum and maximum); asterisk indicates the outliers. The CAP measurements are divided by 100 to reach a homogenous scale (CAP, Controlled attenuation parameter; SWE, Shear wave elastography; TE, Transient elastography).

\begin{tabular}{|c|c|c|c|c|c|c|}
\hline & \multicolumn{2}{|c|}{$\operatorname{Sex}($ Mean $\pm S D)$} & \multirow{2}{*}{ P-value ${ }^{a}$} & \multicolumn{2}{|c|}{ BMI $^{\mathbf{a}}$} & \multirow{2}{*}{ P-value $^{a}$} \\
\hline & Male & Female & & $<\mathbf{3 0}$ & $\geq \mathbf{3 0}$ & \\
\hline TE & $8.75 \pm 8.27$ & $7.21 \pm 5.87$ & 0.389 & $11.14 \pm 11.92$ & $7.1 \pm 1.21$ & 0.235 \\
\hline SWE & $8.7 \pm 8.27$ & $7.11 \pm 5.37$ & 0.34 & $10.78 \pm 11.34$ & $7.12 \pm 4.64$ & 0.258 \\
\hline CAP & $263.21 \pm 63.69$ & $274.86 \pm 72.07$ & 0.502 & $310.21 \pm 71.85$ & $257.1 \pm 61.2$ & $0.005^{b}$ \\
\hline B-mode ratio & $1.46 \pm 0.46$ & $1.58 \pm 0.5$ & 0.298 & $1.72 \pm 0.55$ & $1.46 \pm 0.44$ & 0.118 \\
\hline
\end{tabular}

Abbreviations: CAP, controlled attenuation parameter; SWE, shear wave elastography; TE, transient elastography; BMI, body mass index; SD, standard deviation.

${ }^{a}$ Independent $t$-test

${ }^{\mathrm{b}}$ Mann-Whitney test

by age or sex. It should be noted that several studies have reported older age as a confounding factor for TE and SWE (29-31). In the current study, most elderly patients were excluded due to inadequate cooperation for performing the tests; therefore, the study was performed on a relatively young population with the median age of 47 years.

The majority of published studies support the higher accuracy of non-invasive methods in the detection of more advanced fibrosis (32-35). The present study mostly included patients with non-advanced stages of fibrosis, with more than $80 \%$ of patients having stage 2 fibrosis or lower. The main reason is that sonographic features of cirrhosis and the presence of ascites were among the exclusion cri- teria of this study. Therefore, further studies on a larger number of patients with hepatic fibrosis stages 3 and 4 are needed to assess the correlation of these two modalities in advanced stages of fibrosis. Finally, the recommended cutoff values for fibrosis staging by TE showed moderate concordance with SWE, and they should not be used interchangeably. It is recommended that future studies use liver biopsy as the gold standard reference to assess the internal validity, sensitivity, and specificity of both modalities and the SWE cutoff points. 
A

TE
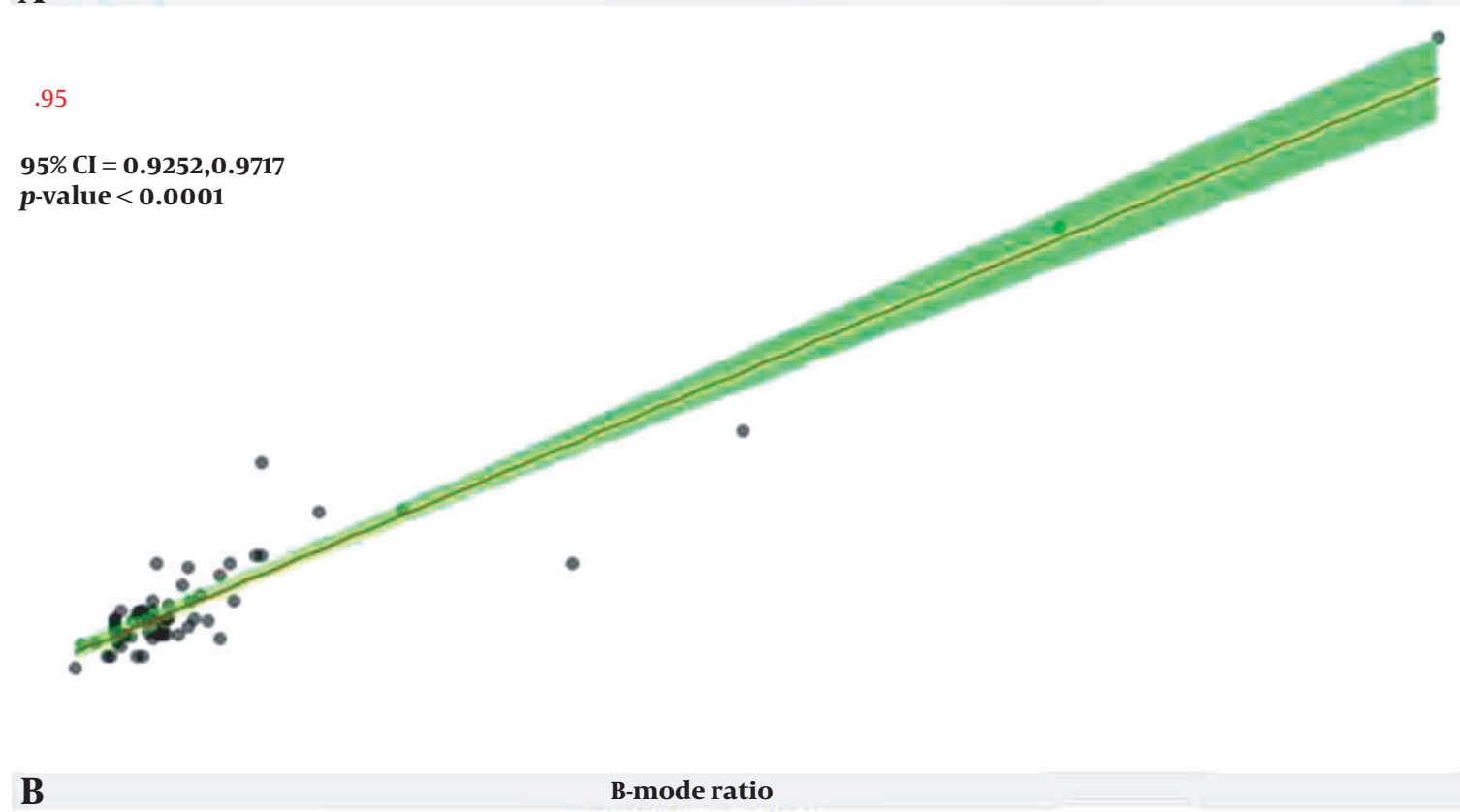

.43

95\% CI $=0.2048,0.6104$

$p$-value $<0.0001$

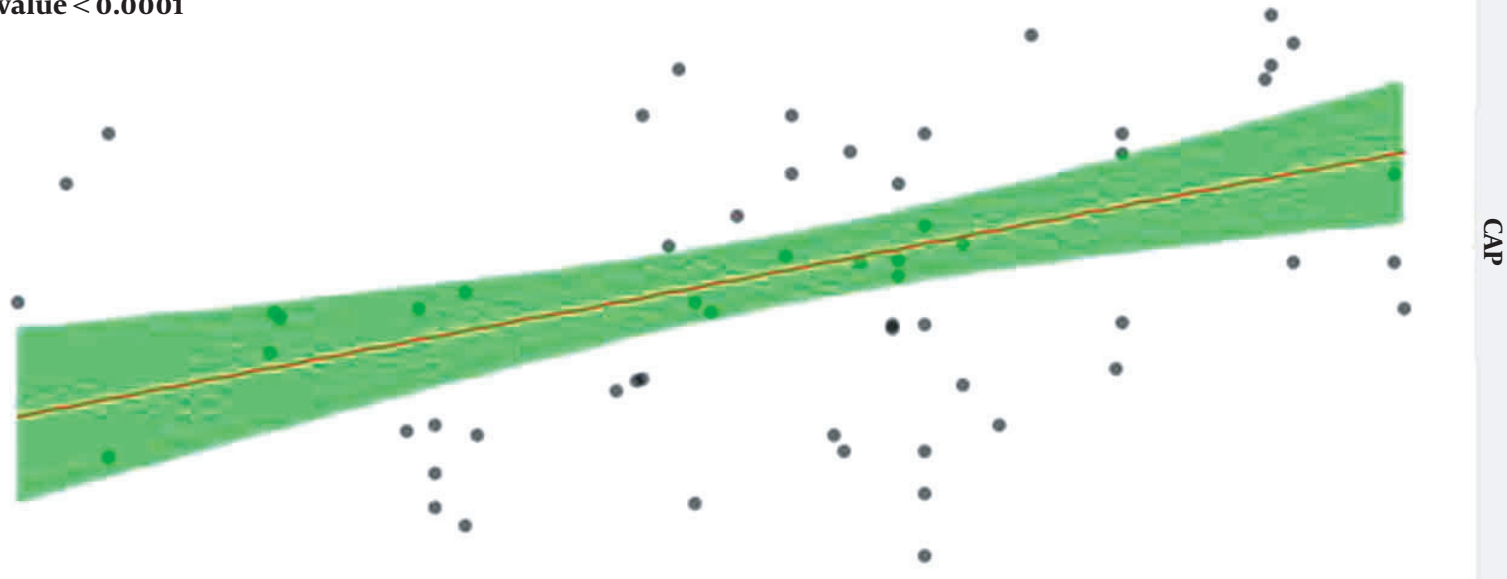

Figure 2. Linear correlations (A) between TE and SWE and (B) between CAP and B-mode ratio (each dot is representative of a case; the green area represents 95\% confidence interval [CIs]). Red numbers represent the Pearson's correlation coefficients. CAP, Controlled attenuation parameter; SWE, Shear wave elastography; TE, Transient elastography.

\subsection{Hepatosteatosis}

Gray-scale ultrasound is commonly used for hepatosteatosis screening. However, it has some limitations, such as interference of liver fibrosis and tissue inflammation with the accuracy of results. Another limitation of gray-scale ultrasound is a low sensitivity of about 55\% for lower grades of steatosis. Also, high BMI and subcutaneous fat content cause technical difficulties and signal attenu- ation, resulting in decreased accuracy (12, 36-38). Besides, this modality is a subjective, operator-dependent visual tool with interobserver variability (39). Accordingly, more reliable non-invasive techniques are essential for a precise assessment of hepatosteatosis and its severity. The B-mode hepatorenal ratio and CAP are new parameters for the measurement of hepatostaosis, with possibly higher accuracies than gray-scale ultrasound. 
Not many studies have compared the CAP and B-mode ratio. In a study by Fujiwara et al. (40), similar to our findings, the correlation between these parameters was high $(r=0.81)$. In the present study, almost two-thirds of the patients were classified into moderate or severe hepatosteatosis groups, based on all three methods. It seems that the B-mode ratio method can detect minimal steatosis more efficiently than other methods, as mild steatosis was detected in a larger number of patients compared to gray-scale sonography or CAP. Therefore, the B-mode ratio can be the preferred option for the early detection of fatty liver.

There are also fewer findings supporting the superiority of the B-mode ratio over other methods. In the present study, none of the studied demographic variables influenced the B-mode ratio measurements, while BMI interacted with the CAP measurements. In the B-mode ratio method, we can map the liver tissue, whereas the CAP method is limited due to the interference of extrahepatic structures with the measurements because of a fixed ROI. Additionally, the B-mode ratio can be measured by a single probe (B-mode probe), while for CAP measurements in obese cases, it may be essential to replace the M-probe with an XL probe $(41,42)$.

In conclusion, similar to previous research, noninvasive modalities were strongly correlated for the assessment of fibrosis and hepatosteatosis in the present study. It was found that factors, such as age, sex, and BMI, did not interfere with the tests results, although BMI was a confounding factor for the CAP results. Overall, selection of the best non-invasive method for the assessment of hepatic fibrosis and steatosis depends on factors other than internal validity. In a local setting, SWE and B-mode ratio measurement may be preferred for detecting fibrosis and hepatosteatosis, respectively.

The limitation of this study was the lack of histopathological results of liver biopsies as a gold standard for comparison. Future studies are recommended to use liver histology as a gold standard to compare the diagnostic results of different hepatic fibrosis and steatosis grading tools.

\section{Footnotes}

Authors' Contribution: Study conception and design: Fariba Zarei and Maryam Moini; acquisition of data: Ravanfar Haghighi and Mahsa Abedi; analysis and interpretation of data: Banafsheh Zeinali-Rafsanjani, Fariba Zarei, and Mahsa Abedi; drafting of the manuscript: Ravanfar Haghighi and Banafsheh Zeinali-Rafsanjani; critical revision of the manuscript for important intellectual content: Banafsheh Zeinali-Rafsanjani and Mahsa Abedi; statistical analysis: Banafsheh Zeinali-Rafsanjani; and study administration and supervision: Fariba Zarei and Maryam Moini.

Conflict of Interests: There is no conflict of interest.

Ethical Approval: This study was approved by the Faculty Research Ethics Committee of Shiraz University of Medical Sciences (ethics code: IR.sums.med.rec.1397.454).

Funding/Support: There was no funding support for this study.

Informed Consent: Written consent was obtained from each participant before enrollment in the study.

\section{References}

1. Younossi ZM, Koenig AB, Abdelatif D, Fazel Y, Henry L, Wymer M Global epidemiology of nonalcoholic fatty liver disease-Meta-analytic assessment of prevalence, incidence, and outcomes. Hepatology. 2016;64(1):73-84. doi: 10.1002/hep.28431. [PubMed: 26707365].

2. Wong RJ, Aguilar M, Cheung R, Perumpail RB, Harrison SA, Younossi $\mathrm{ZM}$, et al. Nonalcoholic steatohepatitis is the second leading etiology of liver disease among adults awaiting liver transplantation in the United States. Gastroenterology. 2015;148(3):547-55. doi 10.1053/j.gastro.2014.11.039. [PubMed: 25461851].

3. Gines P, Cardenas A, Arroyo V, Rodes J. Management of cirrhosis and ascites. N Engl J Med. 2004;350(16):1646-54. doi: 10.1056/NEJMra035021. [PubMed: 15084697].

4. Schwimmer JB, Deutsch R, Kahen T, Lavine JE, Stanley C, Behling C. Prevalence of fatty liver in children and adolescents. Pediatrics 2006;118(4):1388-93. doi: 10.1542/peds.2006-1212. [PubMed: 17015527].

5. Adams LA, Lymp JF, St Sauver J, Sanderson SO, Lindor KD, Feldstein A, et al. The natural history of nonalcoholic fatty liver disease: a population-based cohort study. Gastroenterology. 2005;129(1):113-21. doi:10.1053/j.gastro.2005.04.014. [PubMed:16012941].

6. Bataller R, Brenner DA. Hepatic stellate cells as a target for the treatment of liver fibrosis. Semin Liver Dis. 2001;21(3):437-51. doi: 10.1055/s2001-17558. [PubMed: 11586471].

7. Afdhal NH, Nunes D. Evaluation of liver fibrosis: a concise review. Am J Gastroenterol.2004;99(6):1160-74. doi: 10.1111/j.1572-0241.2004.30110.x. [PubMed: 15180741].

8. Thampanitchawong P, Piratvisuth T. Liver biopsy:complications and risk factors. World J Gastroenterol. 1999;5(4):301-4. doi: 10.3748/wjg.v5.i4.301. [PubMed: 11819452]. [PubMed Central: PMC4695539].

9. Sandrin L, Fourquet B, Hasquenoph JM, Yon S, Fournier C, Mal F, et al. Transient elastography: a new noninvasive method for assessment of hepatic fibrosis. Ultrasound Med Biol. 2003;29(12):1705-13. doi 10.1016/j.ultrasmedbio.2003.07.001. [PubMed:14698338].

10. Sarvazyan A, Hall TJ, Urban MW, Fatemi M, Aglyamov SR, Garra BS. An Overview of Elastography - an Emerging Branch of Medical Imaging. Curr Med Imaging Rev. 2011;7(4):255-82. doi: 10.2174/157340511798038684. [PubMed: 22308105]. [PubMed Central: PMC3269947]

11. Ratziu V, Giral P, Charlotte F, Bruckert E, Thibault V, Theodorou I, et al. Liver fibrosis in overweight patients. Gastroenterology. 2000;118(6):1117-23. doi:10.1016/s0016-5085(00)70364-7.

12. Webb M, Yeshua H, Zelber-Sagi S, Santo E, Brazowski E, Halpern Z, et al. Diagnostic value of a computerized hepatorenal index for sonographic quantification of liver steatosis. AJR Am J Roentgenol. 2009;192(4):909-14. doi:10.2214/AJR.07.4016. [PubMed: 19304694].

13. Crossan C, Tsochatzis EA, Longworth L, Gurusamy K, Davidson B, Rodriguez-Peralvarez $M$, et al. Cost-effectiveness of non-invasive 
methods for assessment and monitoring of liver fibrosis and cirrhosis in patients with chronic liver disease: systematic review and economic evaluation. Health Technol Assess. 2015;19(9):1-409. vvi. doi: 10.3310/hta19090. [PubMed: 25633908]. [PubMed Central: PMC4781028].

14. Bende F, Sporea I, Sirli R, Popescu A, Mare R, Miutescu B, et al. Performance of 2D-SWE.GE for predicting different stages of liver fibrosis using Transient Elastography as the reference method. Med Ultrason. 2017;19(2):143-9. doi: 10.11152/mu-910. [PubMed: 28440347].

15. Kircheis G, Sagir A, Vogt C, Vom Dahl S, Kubitz R, Haussinger D. Evaluation of acoustic radiation force impulse imaging for determination of liver stiffness using transient elastography as a reference. World J Gastroenterol. 2012;18(10):1077-84. doi:10.3748/wjg.v18.i10.1077. [PubMed: 22416182]. [PubMed Central: PMC3296981].

16. Zeng J, Zheng J, Huang Z, Chen S, Liu J, Wu T, et al. Comparison of 2-D Shear Wave Elastography and Transient Elastography for Assessing Liver Fibrosis in Chronic Hepatitis B. Ultrasound Med Biol. 2017;43(8):1563-70. doi: 10.1016/j.ultrasmedbio.2017.03.014. [PubMed: 28483579].

17. Paul SB, Das P, Mahanta M, Sreenivas V, Kedia S, Kalra N, et al. Assessment of liver fibrosis in chronic hepatitis: comparison of shear wave elastography and transient elastography. Abdom Radiol (NY). 2017;42(12):2864-73. doi:10.1007/s00261-017-1213-5. [PubMed 28643136].

18. Thiele M, Detlefsen S, Sevelsted Moller L, Madsen BS, Fuglsang Hansen J, Fialla AD, et al. Transient and 2-Dimensional ShearWave Elastography Provide Comparable Assessment of Alcoholic Liver Fibrosis and Cirrhosis. Gastroenterology. 2016;150(1):123-33. doi: 10.1053/j.gastro.2015.09.040. [PubMed: 26435270].

19. Bota S, Herkner H, Sporea I, Salzl P, Sirli R, Neghina AM, et al. Metaanalysis: ARFI elastography versus transient elastography for the evaluation of liver fibrosis. Liver Int. 2013;33(8):1138-47. doi:10.1111/liv.12240. [PubMed: 23859217].

20. Lupşor M, Badea R, Stefănescu H, Grigorescu M, Serban A, Radu C, et al. Performance of unidimensional transient elastography in staging non-alcoholic steatohepatitis. J Gastrointestin Liver Dis. 2010;19(1):5360.

21. Cournane S, Browne JE, Fagan AJ. The effects of fatty deposits on the accuracy of the Fibroscan(R) liver transient elastography ultrasound system. Phys Med Biol. 2012;57(12):3901-14. doi: 10.1088/0031 9155/57/12/3901. [PubMed: 22643042].

22. Palmeri ML, Wang MH, Rouze NC, Abdelmalek MF, Guy CD, Moser $\mathrm{B}$, et al. Noninvasive evaluation of hepatic fibrosis using acoustic radiation force-based shear stiffness in patients with nonalcoholic fatty liver disease. J Hepatol. 2011;55(3):666-72. doi: 10.1016/j.jhep.2010.12.019. [PubMed: 21256907]. [PubMed Central: PMC3092839].

23. Friedrich-Rust M, Romen D, Vermehren J, Kriener S, Sadet D, Herrmann E, et al. Acoustic radiation force impulse-imaging and transient elastography for non-invasive assessment of liver fibrosis and steatosis in NAFLD. Eur J Radiol. 2012;81(3):e325-31. doi: 10.1016/j.ejrad.2011.10.029. [PubMed: 22119555].

24. Bota S, Sporea I, Sirli R, Popescu A, Jurchis A. Factors which influence the accuracy of acoustic radiation force impulse (ARFI) elastography for the diagnosis of liver fibrosis in patients with chronic hepatitis C. Ultrasound Med Biol. 2013;39(3):407-12. doi: 10.1016/j.ultrasmedbio.2012.09.017. [PubMed: 23245820].

25. Ferraioli G, Tinelli C, Dal Bello B, Zicchetti M, Filice G, Filice C, et al. Accuracy of real-time shear wave elastography for assessing liver fibrosis in chronic hepatitis C: a pilot study. Hepatology. 2012;56(6):2125-33. doi: 10.1002/hep.25936. [PubMed: 22767302].

26. de Lédinghen V, Vergniol J. Transient elastography (FibroScan). Gastroenterol Clin Biol. 2008;32(6):58-67. doi: 10.1016/s03998320(08)73994-0.

27. Cassinotto C, Boursier J, de Ledinghen V, Lebigot J, Lapuyade B, Cales $P$, et al. Liver stiffness in nonalcoholic fatty liver disease: $A$ comparison of supersonic shear imaging, FibroScan, and ARFI with liver biopsy. Hepatology. 2016;63(6):1817-27. doi: 10.1002/hep.28394. [PubMed: 26659452].

28. Cassinotto C, Lapuyade B, Mouries A, Hiriart JB, Vergniol J, Gaye D, et al. Non-invasive assessment of liver fibrosis with impulse elastography: comparison of Supersonic Shear Imaging with ARFI and FibroScan(R). J Hepatol. 2014;61(3):550-7. doi: 10.1016/j.jhep.2014.04.044. [PubMed: 24815876].

29. Sporea I, Bota S, Gradinaru-Tascau O, Sirli R, Popescu A, Jurchis A. Which are the cut-off values of 2D-Shear Wave Elastography (2DSWE) liver stiffness measurements predicting different stages of liver fibrosis, considering Transient Elastography (TE) as the reference method? Eur J Radiol.2014;83(3):e118-22. doi:10.1016/j.ejrad.2013.12.011. [PubMed: 24380640].

30. Castera L, Foucher J, Bernard PH, Carvalho F, Allaix D, Merrouche W, et al. Pitfalls of liver stiffness measurement: a 5-year prospective study of 13,369 examinations. Hepatology. 2010;51(3):828-35. doi: 10.1002/hep.23425. [PubMed: 20063276].

31. Sirli R, Sporea I, Bota S, Jurchis A. Factors influencing reliability of liver stiffness measurements using transient elastography (Mprobe)-monocentric experience. Eur J Radiol. 2013;82(8):e313-6. doi: 10.1016/j.ejrad.2013.03.002. [PubMed: 23562532].

32. Nierhoff J, Chavez Ortiz AA, Herrmann E, Zeuzem S, Friedrich-Rust M. The efficiency of acoustic radiation force impulse imaging for the staging of liver fibrosis: a meta-analysis. Eur Radiol. 2013;23(11):304053. doi: 10.1007/s00330-013-2927-6. [PubMed: 23801420].

33. Friedrich-Rust M, Nierhoff J, Lupsor M, Sporea I, FierbinteanuBraticevici C, Strobel D, et al. Performance of Acoustic Radiation Force Impulse imaging for the staging of liver fibrosis: a pooled meta-analysis. JViral Hepat. 2012;19(2):e212-9. doi: 10.1111/j.13652893.2011.01537.x. [PubMed: 22239521].

34. Guerra JA, Trippia M, Pissaia A, Teixeira BC, Ivantes CA. Acoustic Radiation Force Impulse Is Equivalent to Liver Biopsy to Evaluate Liver Fibrosis in Patients with Chronic Hepatitis C and Nonalcoholic Fatty Liver Disease. Arq Gastroenterol. 2015;52(3):234-8. doi: 10.1590/S000428032015000300015. [PubMed: 26486293].

35. Silva Junior RG, Schmillevitch J, Nascimento Mde F, Miranda ML, Brant PE, Schulz PO, et al. Acoustic radiation force impulse elastography and serum fibrosis markers in chronic hepatitis C. Scand J Gastroenterol. 2014;49(8):986-92. doi: 10.3109/00365521.2014.909528. [PubMed: 24742130].

36. Sasso M, Miette V, Sandrin L, Beaugrand M. The controlled attenuation parameter (CAP): a novel tool for the non-invasive evaluation of steatosis using Fibroscan. Clin Res Hepatol Gastroenterol. 2012;36(1):1320. doi: 10.1016/j.clinre.2011.08.001. [PubMed: 21920839].

37. Fishbein M, Castro F, Cheruku S, Jain S, Webb B, Gleason T, et al. Hepatic MRI for fat quantitation: its relationship to fat morphology, diagnosis, and ultrasound. J Clin Gastroenterol. 2005;39(7):619-25. doi: 10.1097/00004836-200508000-00012. [PubMed:16000931].

38. Albrecht T, Blomley MJ, Cosgrove DO, Taylor-Robinson SD, Jayaram $\mathrm{V}$, Eckersley R, et al. Non-invasive diagnosis of hepatic cirrhosis by transit-time analysis of an ultrasound contrast agent. The Lancet. 1999;353(9164):1579-83. doi: 10.1016/s0140-6736(98)06373-9.

39. Strauss S, Gavish E, Gottlieb P, Katsnelson L. Interobserver and intraobserver variability in the sonographic assessment of fatty liver. AJR Am J Roentgenol. 2007;189(6):W320-3. doi: 10.2214/AJR.07.2123. [PubMed: 18029843].

40. Fujiwara Y, Kuroda H, Abe T, Ishida K, Oguri T, Noguchi S, et al. The B-Mode Image-Guided Ultrasound Attenuation Parameter Accurately Detects Hepatic Steatosis in Chronic Liver Disease. Ultrasound Med Biol. 2018;44(11):2223-32. doi: 10.1016/j.ultrasmedbio.2018.06.017. [PubMed: 30077415].

41. Karlas T, Petroff D, Sasso M, Fan JG, Mi YQ, de Ledinghen V, et al. Individual patient data meta-analysis of controlled attenuation parameter (CAP) technology for assessing steatosis.J Hepatol. 2017;66(5):1022-30. 
doi: 10.1016/j.jhep.2016.12.022. [PubMed: 28039099].

42. Sasso M, Audiere S, Kemgang A, Gaouar F, Corpechot C, Chazouilleres $\mathrm{O}$, et al. Liver Steatosis Assessed by Controlled Attenuation Parame- ter (CAP) Measured with the XL Probe of the FibroScan: A Pilot Study Assessing Diagnostic Accuracy. Ultrasound Med Biol. 2016;42(1):92-103. doi: 10.1016/j.ultrasmedbio.2015.08.008. [PubMed: 26386476]. 